\title{
Reverse-High Performance Liquid Chromatography Mechanism Explained by Polarization of Stationary Phase
}

\author{
Auréa Andrade-Eiroa*, Tanh Le-Cong, Mai-Lan Nguyen and Philippe Dagaut \\ CNRS-ICARE, 1C, Avenue de la Recherche Scientifique - 45071 Orléans Cedex 2, France. \\ *Corresponding author: eiroa_2000@yahoo.es, andrade@cnrs-orleans.fr, Tel. (+33) 238 255466, Fax. (+33) \\ 238696004 \\ doi:10.5618/chem.2011.v1.n1.8 || Received: 14-10-2011, Accepted: 28-10-2011, Available online: 13-11-2011
}

\begin{abstract}
Original results and conclusions on Reverse Phase High Performance Liquid Chromatography (RPLC) mechanism are here presented. So far, none of the theoretical approaches applied to the RPLC mechanism can explain the retention and elution mechanisms of most of the analytes by RPLC, especially neutral organic compounds. Our experiences allowed us to state that RPLC retention mechanism most likely occurs through polarization of stationary phase (usually dielectric surfaces) submerged into solvents with huge dielectric constant and high dipole moment (i.e. water and/or acetonitrile) at high pressures as those applied in RPLC systems. The surface polarized interacts with polarizable target analytes (i.e. naphthalene, pyrene or benzo(ghi)perylene) in such a way that the retention depends on the medium polarizability of the target compound. The higher is the medium polarizability of the compound retained the higher is its retention time.
\end{abstract}

By using Polycyclic Aromatic Hydro-carbons (PAHs) and 6-nitrochrysene as probes, we found that although the dependence of retention times on pressure is not evident in most cases, the interaction between pressure and the mobile phase dipole moment is evidenced at the light of the constructed mathematical models. Two very different cases can be distinguished:

1. On one hand, when low dipole moment mobile phases are used, high pressure means big work developed by the system in such a way that high pressures inside the system reduce retention times compared to low pressures.

2. On the another one, when high dipole moment solvents are used as mobile phase, high pressure entails an outstanding increase of the retention times compared to low dipole moment mobile phases. This is most likely due to high pressures that entail closer alignment of mobile phase dipoles and lesser tilt of them, originating an outstanding electric field inside the analytical column.
In addition to this, reduced retention times for dipole solutes (6-nitrochrysene, dipole molecule) compared to non-polar solutes (PAHs) were observed when pressure drops. This conclusions would allow to extend the model to basic/acid molecules at different $\mathrm{pH}$. In this case, electrostatic repulsions among analyte molecules should be considered.

Keywords: Reverse High Performance Liquid Chromatography mechanism; $\mathrm{PAH}$; nitro-PAH; theory; stationary phase; dielectrics; polarization.

\section{Introduction}

Reversed-Phase high-performance liquid chromatography (RPLC) has become one of the most powerful and often used techniques for the resolution of mixtures, identification, and quantification of organic compounds in all kind of samples with or without previous clean up steps [1]. However, the real mechanism of RPLC retention is yet not fully understood [2-4]. Up to now, whether solutes show an adsorptive behavior on the chemically bonded phases or if a partitioning mechanism occurs is still an open question [5-8].

The solvophobic theory, proposed in 1976 [9] provided the first rigorous treatment of the retention energetics in RPLC. This theory is based on a thermodynamic cycle and views the retention in RPLC as association in vacuo between the elute and the stationary phase and subsequent transfer of the participating species into the mobile phase $[8,9]$. This model has been considered very useful at predicting trends in retention upon changes in mobile phase composition and in altering certain molecular properties of the solute, principally its size [7]. However, it underestimates effects of cavity formation in the stationary phase $[10,11]$ and it does not take into account possible solute-stationary phase interactions or the experimentally supported fact that retention depends on the nature of the bonded chain phase [12].

Another very popular theory is the lipophilic theory of Carr and co-workers [7]. According to this theory, 
favorable interactions between the solute and the stationary phase drive transfer from mobile to the stationary phase. However, recent papers have demonstrated that neither the solvophobic theory advocated by Horváth and co-workers nor the lipophilic advocated by Carr and co-workers can adequately describe the mechanisms in actual RPLC systems [13]. As a matter of fact, results of modern investigations have proved that both non polar and polar solutes have favorable interactions with both the mobile and the stationary phase thus showing that neither the solvophobic nor the lipophilic theory alone can explain the driving forces for retention in RPLC [14].

Currently, papers consider simultaneous contributions from more than one retention mechanism: adsorption on free silanols and partitioning into the chemically bonded phase [15], with preponderance of a mechanism that is largely partitioning, particularly for $\mathrm{C}_{18}$ stationary phases and for small non-polar solutes $[3,16,17,18,19]$. Examples of this kind of theories are the unified molecular theory model of Martire and Boehm [16] or the statistical-mechanical theory [10]. Both of these theories consider that retention energies are dominated by partitioning of eluates into the bonded-phase, although monomolecular layer cannot be considered a phase in classical thermodynamics, and although they cannot explain why retention of analytes on the stationary phase changes when solid phase particles become saturated by organic modifier [20]. On the other hand, an application of the partitioning theory leads to controversy in the description of some RPLC data [4, 21]. In fact, silica-bonded alkyl ligands have been shown to act more as an anisotropic inter-phase than as a bulk liquid phase in RPLC $[4,6,10]$.

Steric effects as well as thermodynamic considerations were taken into account by approaches such as the "Slot model". This one states that retention is due to penetration of solutes between alkyl chains. Long narrow molecules fit better than square shaped or bulky ones, and are thus retain stronger. This idea had been introduced more formally in Dill's lattice model for retention $[6,10]$. Thermodynamically, a larger cavity must be opened in the stationary phase chains for the non-planar compounds, and this is entropically expensive. Therefore, the non-planar compound should elute first [22]. A second approach was presented by Yan and Martire [23]. Their descriptor states a linear relationship between minimum areas of a box enclosing the molecule and retention times, with smaller values of area of enclosing box resulting in larger retention values.

On the other hand, adsorption approaches try to account for the influence of alkyl chain nature, their surface density, the presence or absence of endcapping of the stationary phases 24]. However these approaches also give rise to contradictory results. Although a correlation between adsorption equilibrium constant $(K)$ and carbon content of stationary phases was demonstrated showing the value of $K$ increases with increasing carbon content, slope of the curves gradually decreases for carbon contents above around $10 \%$ and almost the same values of $K$ are observed at $13.7 \%$ and $17.1 \%$ [25]. Therefore many authors argued that only part of the $\mathrm{C}_{18}$ ligands contributes to the retention behavior in RPLC [26]. Other authors claim that this phenomenon is due to the fact that when more ligands are present inside the pores of silica particles, both pore volume and surface decrease. Hence, the solid/liquid contact interface becomes smaller [27]. However, they cannot explain the very strong retention of large molecules such as benzo[ghi]perylene compared to smaller molecules like naphthalene under the same experimental conditions. On the other hand, reality seems to be more complicated than that. As a matter of fact, for surface coverage larger than $2 \mu \mathrm{mol} / \mathrm{m}^{2}$, the saturation capacities of all types of sites decrease with increasing bonding density. At lower densities, the variation of the saturation capacities with the bonding density is more complicated. It may increase or decrease depending on the nature of the target analyte and the type of adsorption sites considered. Thus, the actual nature of the heterogenous adsorption on RPLC packing material remains unclear [28]. On the other hand, changes in the phase ratios can produce deviations from linearity in the van't Hoff equation, usually applied for studying the thermodynamics of the transfer from the mobile phase to the stationary phase in RPLC [4]. When non-linear van't Hoff plots are observed, it is assumed that enthalpy and entropy change with temperature. However when changes in phase ratios occur, the non-linearity in the van't Hoff equation behavior may or may not be due to changes in the entropy and enthalpy changes [4].

Other controversies are found in reference to adsorption approaches. In fact, Gritti et al. [29, 30] stated that increasing the water concentrations of the mobile phase leads to the increase of the density of the high energy sites on conventional $\mathrm{C}_{18}$-bonded phases. However, it is well-known that in general water content above $90 \%$ in the mobile phase entails the retention on RPLC columns drops drastically. Authors have established that this drastic loss of retention upon increased concentration of water in the mobile phase is due to the collapse of alkyl chains [31]. However, according to recent papers it seems that increasing the water concentration of the mobile phase increases the clustering of the alkyl chains but does not cause necessarily their collapse [29, 30]. Other authors justify this loss of retention (when pure water is used) by de-wetting of the surface [32] although Kazakevich and El'tekov [33] demonstrated several decades ago that the solid surfaces used in RPLC are impermeable 
to liquid solutions used in this type of chromatography. Other authors tried to justify this loss of retention of RPLC columns in presence of water based on the conformational disorder of the alkyl chains in presence of water. But in contrast with this conclusion, Pemberton et al. [34] reported that the Raman spectral results show only little differences in overall conformational order of alkyl chains in water versus other polar organic solvents.

Among the other thermodynamic approaches it is worth pointing out the Linear Free Energy Relationships (LFERs). One of the most relevant LFERs is the Solvation Parameter Model proposed by Abraham [35-38]. This approach tries modeling retention times by a simple mathematical relationship including different solute descriptors: the solute's characteristic volume, its excess molar refraction, the ability of the solute to stabilize a neighboring dipole in virtue of its capacity for orientation and induction interactions, the solute's effective hydrogen-bond acidity, and the solute's effective hydrogen-bond basicity [39]. The main limitation of this approach, as other quantitative retention-structure relationship methodologies, is that it lacks accuracy due to problems in the translation of the properties encoded in the molecular structures to descriptor values [40]. The second limitation of this approach concerns its applicability since the models are usually developed for specific mobile phase compositions. This prevents predictions at any other conditions and makes the comparison among solvents and columns difficult. General solvation model including common parameters for all compounds and mobile phases has the capability of predicting retention times at any solvent composition. However, the accuracy and precision of the predictions get poorer than those achieved using models particularized to each solvent composition [41]. Also, this model fails to predict retention times for some compounds such as benzofuran, biphenyl and dibutyl phthalate [42].

In this work, we found that the retention times of each PAH and 6-nitrochrysene in $\mathrm{C}_{18}$-RPLC can be mathematically modeled by using dipole moments of the mobile phase and pressure inside the column as the only dependent variables. For each PAH and for 6nitrochrysene, similar mathematical relationships capable of predicting their retention times in very different mobile phases were found.

The key of RPLC mechanism might be the polarization of stationary phase into solvents with high dipole moment and extraordinarily high dielectric constant at high pressures as those developed by HPLC systems. The polarization of stationary phase could be due to the spontaneous alignment of mobile phase dipoles on the stationary phase. Due to dielectric constants of liquids and solids are related to their dipole moments [43], from now on only dipole moment will be included in the mathematical models constructed. Differences among the PAHs retention times could be explained through their different molecular polarizabilities because polarizability measures the interaction of the electrons in a molecule with an electric field.

Although apparently there is no relationship between PAHs retention times and pressure, the interaction between dipole moment of the mobile phase and pressure inside the column becomes evident as revealed by the mathematical equations constructed here for the probe molecules retention times.

\section{Experimental and Modeling}

2.1. HPLC System Chromatographic experiments were performed using a high performance liquid (HPLC) system (Shimadzu, Kyoto, Japan). This instrument consisted of: a system controller, a solvent delivery module, an auto-sampler, a column oven and a UV/Vis Photodiode Array Detector, and Spectrofluorimetric Detector RF-10AxL. A Vydac 201TPC 18 $5 \mu \mathrm{m}, 250 \mathrm{~mm}$ length $\mathrm{x} 4.6 \mathrm{~mm}$ inner diameter column (Grace Davison Discovery Sciences, Lokeren, Belgium) was chosen as analytical column. Figure 1 presents the structure of the stationary phase.

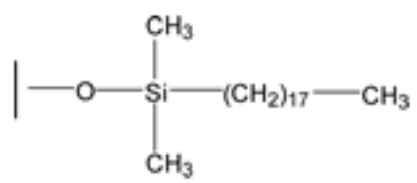

Figure 1. Structure of $\mathrm{C}_{18}$ stationary phase.

2.2. Reagents A mixture of 16 PAHs $(2000 \mu \mathrm{g} / \mathrm{ml}$ each component in 1:1 methylene chloride:benzene from Sigma-Aldrich) was used for preparing standards by dilution. Components in the mixture were: Acenapthene, acenaphthylene, anthracene, benz(a) anthracene, benzo(b)fluoranthene, benzo(k) fluoranthene, benzo(ghi)peyrlene, benzo(a)pyrene, chrysene, dibenzo(ah)anthracene, fluoranthene, fluorene, indeno (1,2,3-cd)pyrene, naphthalene, phenanthrene, and pyrene (Table 1). Standards of PAHs were prepared by diluting this initial solution. Anthracene, Phenanthrene, and 6-nitrochrysene (95\% purity) solids, acetonitrile, water, methanol $(99 \%+)$, and dichloromethane Chromasolv HPLC grade were purchased from Sigma-Aldrich. Cyclohexane, ethanol, and n-hexane for residue analysis (Fluka, Riedel-de Haën) were also used.

2.3. Algorithm used for finding mathematical relationships among retention times of target molecules, dipole moment of the mobile phase and pressure developed by the system. Iterative approach was carried out supposing retention time is a function of dipole moment and pressure, that is $R_{t}=f(\mu, P): \mathfrak{R}^{2} \rightarrow \mathfrak{R}$. Relationship found among retention time $\left(R_{t}\right)$, dipole moment $(\mu)$, and Pressure $(P)$ is given by the mathematical power function (1). 


$$
R_{t}=\mu^{\alpha}\left(P^{\beta}+b\right)+C P+D
$$

By giving randomly initial values of $\alpha, b$ and $C$, we obtained:

$$
R_{t 0}=\mu^{\alpha_{0}}\left(P^{\beta_{0}}+b\right)+C_{0} P+D_{0}
$$

At pressure $P$ and dipole moment $\mu$, the retention time will be $R_{t}$. Minimization of $\left(R_{t 0}-R_{t}\right)^{2}$ by varying values of $\alpha, \beta, b, C$, and $D$ will drive us to an appropriate retention time power function.

\begin{tabular}{|c|c|c|c|}
\hline Structure & PAH & Structure & PAH \\
\hline & Naphtalene & & Benz(a)anthracene \\
\hline & Acenaphtylene & & Chrysene \\
\hline & Acenaphtene & & Benzo(b)fluoranthene \\
\hline & Fluorene & & Benzo(a)pyrene \\
\hline & Anthracene & & Benzo(k)fluoranthene \\
\hline & Phenanthrene & & Benzo(ghi)perylene \\
\hline & Fluoranthene & & Indeno[1,2,3-cd]pyrene \\
\hline & Pyrene & & Dibenzo(a,h)anthracene \\
\hline
\end{tabular}

Table 1. Structure of PAHs.

\section{Results and Discussion}

According to literature, retention and selectivity in RPLC depends on the following variables:

i) Stationary phase. The bonded phase and the density of the surface coverage $[44,45]$ are particularly important. As early as 1990, Men and Marshall [3] reported that the non-polar stationary phase not only acts as a passive solute receptor but also plays an active role in the retention mechanism of RPLC. In addition to this, recent studies demonstrated that the influence of surface coverage dominates over the influence of alkyl chain length on the conformational order of stationary phase materials [44, 45].

ii) The mobile phase. On one hand, the conformational order of alkyl chains bonded to silica particles in RPLC stationary phases seems to depend on the polarity of the mobile phase. At the same time, the conformational order of the aforementioned chains seems to influence the retention and selectivity shape in RPLC. As a consequence, the retention and selecti- vity in RPLC is supposed to be closely related to the solvents used as mobile phase. However, controversy was found regarding the dependence of solvent polarity on the octadecylsilane chains conformational order. As a matter of fact, while Raman Spectroscopy studies showed that the alkyl portions of the stationary phase (monomeric and polymeric bonded phases) are more disordered in low polarity solvents, such as tetrahydrofuran or n-hexane [34], and that polar solvents (i.e. water or acetonitrile) have only a little impact on the conformational order of the octadecylsilane bonded phase, infrared spectroscopy indicated that the conformational disorder increases upon increase of the solvent polarity [40].

Regarding retention in RPLC and its dependence on conformational order, in general, the highest degree of shape selectivity [16, 47, 48] and retention $[49,50]$ are observed for the most ordered stationary phase. However, controversy can also be found with respect to this issue. In fact, although high conformational order seems to entail stronger 
retention [49, 50], and non polar solvents like nhexane provide a full extended conformation [46] smaller retention times are achieve in RPLC by using solvents with lower polarity than those of acetonitrile and water.

Since recent studies based on molecular dynamics simulations indicated that water exhibits a nonmonotonic behavior of polarization as well as an anomalous dielectric response (the non-proportionality of the polarization and the macroscopic electric field) [51], this solvent will not be included in our modeling of retention times.

On the other hand, literature suggested that the dielectric properties (especially dipole moment and dielectric constant, both of them related as we explained in the Introduction Section) of the mobile phase seem to be the most important factor in RPLC retention [52]. In addition to this, polarizability of the target compound depends on the dipole moment of the solvent/mobile phase and increases with dipole moment increasing [53]. This could be one of the reasons for selecting water and acetonitrile as mobile phases in RPLC. As a consequence, the solvents chosen in this paper widely vary in terms of dipole moment and viscosity (this one related to average pressure inside the column). The solvents included in this paper were: acetonitrile, methanol, ethanol, dichloromethane, cyclohexane, and hexane.

Table 2 shows the retention times of several PAHs and 6-nitrochrysene obtained here using different mobile phases ranging from low dipole moments (ethanol, dichloromethane, cyclohexane, and nhexane) to mobile phases with high dipole moments (methanol, acetonitrile). As seen from this Table, acetonitrile and methanol provided similar retention times (both of them have high dipole moment and similar viscosities). Regarding the selectivity, as indicated in Table 2, acetonitrile and methanol showed a very strong capability for discriminating compounds as a function of their molecular polarizability whereas neither dichloromethane nor cyclohexane showed any selectivity. On the other hand, even though PAHs solubility is higher in dichloromethane than in $n$-hexane (Table 3), retention times in both solvents are similar.

iii) The average pressure. A number of studies about dependence of RPLC retention times on pressure at constant temperature have been performed previously [54, 55]. Szabelski et al. [53] found that the retention of insulin greatly increases when the average pressure increases from 47 to 147 bar. The authors justified this dependence based on the shift of the adsorption equilibrium of insulin caused by the pressure inside the column. On the other hand, Chen and $\mathrm{Li}$ (2004) [55] found that the pressure-induced retention of peptides on RPLC is an entropy-favored and enthalpy-unfavored phenomenon and explained the phenomenon by pressure-induced desorption of solvent molecules associated with the stationary phase or with the target analyte. Most of these papers reported an increase in the retention time for increasing pressures in RPLC [56, 57]. However, in the present work, we found that the dependence of retention time on pressure is a bit more complex, and might diminish or increase depending on the molecule probe and on the mobile phase dipole moment, as presented along this section.

We studied the dependence of retention times on pressure using $70 \% \mathrm{ACN} / 20 \%$ Water at speed flow of $0.25 \mathrm{ml} / \mathrm{min}$ for both PAHs and 6-nitrochrysene (Table 4).

Most of neutral compounds separated by HPLC derive from benzene. This is the main reason for chosing PAHs as target compounds. On the other hand, other reasons for selecting PAHs were:

1. Medium polarizability of PAHs family compounds is easily deduced in a qualitative and intuitive way.

2. The findings can be extrapolated to other neutral compounds and even to acid/basic molecules if electrostatic repulsions are considered (especially when they are ionized).

3. On the other hand, polarizabilities of solute molecules can change if strong interactions between solvent and solute exist, or if strong interactions among solute molecules (i.e. ions in solution, especially in concentrated solutions). Consequently, for simplifying the model and avoid confusion of interactions, we chose neutral molecules which hardly interact each other (PAHs molecules) and with solvent molecules (interactions between acetonitrile/water are expected to be small).

4. With elucidation purposes, 6-nitrochrysene (a dipole molecule) was also included. In this case, electrostatic repulsions among analyte molecules (especially at low pressures) make the retention times of this target analyte elutes before. 
Table 2. Retention times of several PAHs (A: Naphtalene; B: Chrysene; C: Indopyrene; D: 6-nitrochrysene) using different conditioning solvents.

\begin{tabular}{|c|c|c|c|c|c|c|c|}
\hline \multirow{2}{*}{$\begin{array}{l}\text { Conditioning } \\
\text { step } \\
\text { (\% solvent) }\end{array}$} & \multirow{2}{*}{$\begin{array}{l}\text { Elution step ( } \% \text { acetonitrile } \\
-\% \text { water in volume) }\end{array}$} & \multirow{2}{*}{$\begin{array}{l}\text { Speed Flow } \\
(\mathrm{ml} / \mathrm{min})\end{array}$} & \multirow{2}{*}{$\begin{array}{l}\text { Dipole Moment } \\
\text { of the solvent } \\
\text { (Debye) [64] }\end{array}$} & \multicolumn{4}{|c|}{ Retention Time in $\min (\mathrm{a})$} \\
\hline & & & & A & $\mathrm{B}$ & $\mathrm{C}$ & $\mathrm{D}$ \\
\hline $100 \%$ acetonitrile & $100 \%$ acetonitrile & 0.5 & 3.44 & 6.50 & 9.65 & 16.80 & 7.30 \\
\hline $100 \%$ methanol & $100 \%$ methanol & 0.5 & 2.87 & 6.40 & 10.0 & 16.80 & 7.83 \\
\hline $100 \%$ ethanol & $100 \%$ ethanol & 0.5 & 1.66 & 5.85 & 6.00 & 6.20 & 6.50 \\
\hline $\begin{array}{l}100 \% \\
\text { dichloromethane }\end{array}$ & $100 \%$ dichloromethane & 0.5 & 1.14 & 5.97 & 6.20 & 6.20 & 6.00 \\
\hline $\begin{array}{l}100 \% \\
\text { cyclohexane }\end{array}$ & $100 \%$ cyclohexane & 0.5 & 0 & 6.05 & 6.10 & 6.10 & 6.30 \\
\hline $100 \%$ hexane & $100 \%$ hexane & 0.5 & 0 & 6.12 & 6.30 & 6.30 & 7.42 \\
\hline
\end{tabular}

Note: (a) $n=3, S D=7 \%$ except for 6-nitrochrysene: $9 \%$.

Table 3. Solubility data for PAHs at $298 \mathrm{~K}$ unless other temperature is indicated [108]

\begin{tabular}{|l|l|l|}
\hline $\begin{array}{l}\text { Compound } \\
\text { (PAH) }\end{array}$ & Solvent & $\begin{array}{l}\text { Solubility in } \\
\text { molar fraction } \\
\text { (Temperature })\end{array}$ \\
\hline Naphthalene & Hexane & 0.1168 \\
\cline { 2 - 3 } & Ethanol & $0.0180(273 \mathrm{~K})$ \\
\cline { 2 - 3 } & Methylbenzene & 0.2920 \\
\cline { 2 - 3 } & 1-Hexanol & $0.0540(282.2 \mathrm{~K})$ \\
\cline { 2 - 3 } & 1-Octanol & $0.0690(280.9 \mathrm{~K})$ \\
\cline { 2 - 3 } & 1-Pentanol & 0.0811 \\
\cline { 2 - 3 } & 1-Propanol & $0.0476(296.1 \mathrm{~K})$ \\
\cline { 2 - 3 } & 2-Butanol & $0.0656(301.9 \mathrm{~K})$ \\
\cline { 2 - 3 } & 2-Propanol & $0.0371(297.6 \mathrm{~K})$ \\
\cline { 2 - 3 } & 2-Propanone & $0.1640(287.5 \mathrm{~K})$ \\
\cline { 2 - 3 } & Benzene & $0.3318(303 \mathrm{~K})$ \\
\cline { 2 - 3 } & Dichloromethane & 0.3300 \\
\cline { 2 - 3 } & n-Octadecane & $0.5224(325.1 \mathrm{~K})$ \\
\cline { 2 - 3 } & Methanol & $0.0255(297.6 \mathrm{~K})$ \\
\hline Phenanthrene & n-Hexane & 0.0326 \\
\cline { 2 - 3 } & Ethanol & $0.0156(307.65 \mathrm{~K})$ \\
\cline { 2 - 3 } & n-Hexane & 0.0326 \\
\cline { 2 - 3 } & Cyclohexane & $0.0606(307.65 \mathrm{~K})$ \\
\cline { 2 - 3 } & Benzene & $0.2875(312.75 \mathrm{~K})$ \\
\hline \multirow{5}{*}{ Anthracene } & Hexane & 0.00150 \\
\cline { 2 - 3 } & Methanol & 0.00477 \\
\cline { 2 - 3 } & Methylbenzene & 0.00736 \\
\cline { 2 - 3 } & 1-Butanol & 0.000801 \\
\cline { 2 - 3 } & 1-Octanol & 0.002160 \\
\cline { 2 - 3 } & 2-Propanol & 0.000411 \\
\cline { 2 - 3 } & Benzene & 0.007418 \\
\cline { 2 - 3 } & Methanol & 0.000202 \\
\cline { 2 - 3 } & n-Hexane & 0.001290 \\
\cline { 2 - 3 } & n-Octadecane & $0.5577(466.5 \mathrm{~K})$ \\
\cline { 2 - 3 } & Acetonitrile & $0.000556(\mathrm{ca} .296$ \\
$\mathrm{K})$
\end{tabular}

\begin{tabular}{|l|l|l|}
\hline Pyrene & Hexane & $0.00852(299 \mathrm{~K})$ \\
\cline { 2 - 3 } & Methanol & $0.0673(296 \mathrm{~K})$ \\
\cline { 2 - 3 } & Methylbenzene & $0.0567(293 \mathrm{~K})$ \\
\cline { 2 - 3 } & n-Octadecane & $0.6061(407.0 \mathrm{~K})$ \\
\cline { 2 - 3 } Acetonitrile & $0.00574(294 \mathrm{~K})$ \\
\cline { 2 - 3 } 2-Butanol & $0.00433(324 \mathrm{~K})$ \\
\cline { 2 - 3 } & 2-Propanone & $0.0284(293 \mathrm{~K})$ \\
\cline { 2 - 3 } & Ethanol & $0.00288(293 \mathrm{~K})$ \\
\cline { 2 - 3 } & Methylbenzene & $0.0354(296 \mathrm{~K})$ \\
\cline { 2 - 3 } & Methylbenzene & $0.00204(294 \mathrm{~K})$ \\
\cline { 2 - 3 } & n-Heptane & $0.0012(293 \mathrm{~K})$ \\
\cline { 2 - 3 } & 2-Propanone & $0.000634(294 \mathrm{~K})$ \\
\cline { 2 - 3 } & Cyclohexane & $0.000149(294 \mathrm{~K})$ \\
\hline
\end{tabular}

Table 4. Dependence of 6-nitrochrysene retention times on pressure in HPLC column (silica- $\mathrm{C}_{18}$ stationary phase, $70 \% \mathrm{ACN}$ in conditioning and elution steps).

\begin{tabular}{|l|l|l|}
\hline $\begin{array}{l}\text { Speed Flow } \\
(\mathrm{ml} / \mathrm{min})\end{array}$ & Pressure (bar) & $\begin{array}{l}\text { Retention Time (min) } \\
(\mathrm{n}=3, \mathrm{SD} 7 \%)\end{array}$ \\
\hline 0.25 & 10 & 17.6 \\
\hline 0.25 & 19 & 39.0 \\
\hline 0.25 & 21 & 42.6 \\
\hline 0.25 & 65 & 46.9 \\
\hline 0.25 & 89 & 51.0 \\
\hline
\end{tabular}

A pressure control manifold was used in this case for controlling the pressure inside the analytical column. Pressures ranged from 10 to 89 bar (Table 4). We did not find significant influence of pressure on PAHs retention times. However, significant influence of pressure on 6-nitrochrysene retention times was observed. As we explained in the Introduction Section, our results suggest the existence of electric 
field inside the analytical column most likely created by spontaneous alignment of dipoles on the stationary phase at high pressures as it was explained in the Introduction section. It is possible that dipole molecules attract each other inside the column in presence of the electric field, but when this one becomes weak (especially if pressure drops too much), repulsion among dipole molecules occurs and retention times shorten. In fact, Avdeenkov and Bohn [58] proposed a theory about a new molecular state for gases at very low temperatures. Two gaseous polar molecules at extremely low temperatures and in presence of an electric field can form a weak link giving a super molecule due to considerable intermolecular interactions. Both polar molecules act like compass needles, which are small bar magnets. When the needles are far apart, both point north, but as they approach, the magnetic dipole forces between them takes over and causes the needles to attract and align head-to-tail [59]. When two head-to-tail-aligned molecules approach, it is possible that one will flip its orientation and create repulsion. If the molecules begin to move apart, one might flip again, creating an attraction. In the presence of an electric field, there is a specific distance where these flips can occur, i.e. where the attraction and repulsion energies are equal. The properties of the field-linked state, including its binding energy and the distance between the molecules, depend entirely on the strength of the electric field. Extension or application of this theory to molecules in solution would lead us to the conclusion that dipoles in presence of an electric field undergo attraction and this attraction disappears when electric field becomes weak or disappears.

On the other hand, although Loeser [60] concluded that shorter retention times for dipoles can be explained by the negative potential of silica derivatized column, we must remind that a negative potential not always mean negative net (global) charge. In fact, negative potential means a dipole oriented with the negative side towards the analyzed surface [61].

As for pressure developed by the RPLC system, this is related to viscosity of the solvent (at a given flow rate, the higher the viscosity, the higher the pressure inside the column) and to the temperature (the higher the temperature, the lower the solvent viscosity and consequently the pressure). Therefore, variations of the average pressure inside the column were obtained by changing the solvent so that the interaction between the dipole moment of the mobile phase and pressure could be simultaneously studied.

Concerning pressure dropping along the column, in general losses of pressures can be also neglected since HPLC devices are designed for avoiding large losses of pressures along the column. iv) The target analyte. As can be seen from Table 6, our results indicated retention times increase with increasing ring-number in the PAHs. For 6nitrochrysene the retention times were lower than for its homologue chrysene in almost all the solvents considered, although more interactions between stationary phase and this target analyte and among analyte molecules are expected in the case of 6nitrochrysene. Several authors have pointed out that the elution order in RPLC columns depends on the medium polarizability of the analytes $[62,63]$. This could explain why, in general, higher retention times are observed for higher ring PAHs (i.e. for chrysene and indeno(1,2,3-cd)pyrene), compared to naphthalene, and why asymmetric PAHs are stronger retained than symmetric PAHs (chrysene vs. pyrene for example) but the data for 6-nitrochrysene cannot be clearly interpreted.

v) Conditioning of the analytical column. In the present experiments (Table 5), the conditioning of the column influences the elution times of the probe molecules. The results showed the effect of mobile phase on the retention times: the higher the percentage of water used during the conditioning step, the longer is the retention time. In fact, as we can see from Table 2, the retention time of naphthalene increases outstandingly upon increasing the water percentage in the mobile phase. A comparison of retention times for chrysene when conditioning was carried out with $20 \%$ acetonitrile $/ 80 \%$ water and elution with $100 \%$ acetonitrile and that when conditioning and elution were made at $100 \%$ acetonitrile confirms this conclusion. In the first case, the retention time was $11.37 \mathrm{~min}$ whereas it was $9.65 \mathrm{~min}$ in the second one. Similar trends were found for the other PAHs and for 6-nitrochrysene. One exception was observed in the case of 6-nitrochrysene when the column is conditioned with $100 \%$ acetonitrile and elution is carried out with $20 \%$ acetonitrile/ $80 \%$ water. In this case, very long retention times were obtained, probably due to the not intended conditioning effect of water during running chromatogram step before elution of 6-nitrochrysene. The data indicated that 6nitrochrysene seems to be more affected by changes in conditioning step than PAHs. Although authors suggest that this would be the consequence of absorption of water by the silica particle, previous studies performed in our group suggested that most likely $\mathrm{C}_{18}$ bound to silica particles do not absorb water. As a matter of fact, no interactions between the mobile phase gradient and other parameters such as temperature or speed flow were found [63]. This finding suggests that the stationary phase nature does not change upon changes in the mobile phase as proposed earlier [63]. 
Table 5. Experimental retention times for several PAHs and 6-nitrochrysene in RPLC in function of percentage of water in conditioning and elution steps.

\begin{tabular}{|c|c|c|c|c|c|c|}
\hline \multirow{2}{*}{$\begin{array}{l}\text { Conditioning step } \\
\text { (\% acétonitrile - } \\
\% \text { water) }\end{array}$} & \multirow{2}{*}{$\begin{array}{l}\text { Elution step }(\% \\
\text { acetonitrile } \quad-\% \\
\text { water) }\end{array}$} & \multirow{2}{*}{$\begin{array}{l}\text { Speed } \\
\text { Flow } \\
(\mathrm{ml} / \mathrm{min})\end{array}$} & \multicolumn{4}{|c|}{ Retention Time in $\min (\mathrm{a})$} \\
\hline & & & $\begin{array}{l}\text { PAHs } \\
\text { (naphthalene) }\end{array}$ & Chrysene & $\begin{array}{l}\text { Indeno(1,2,3-cd) } \\
\text { Pyrene }\end{array}$ & $\begin{array}{l}\text { 6- } \\
\text { nitrochrysene }\end{array}$ \\
\hline 100 & $70-30$ & 0.5 & 6.34 & 10.00 & 29.50 & 7.20 \\
\hline 100 & $80-20$ & 0.5 & 6.42 & 9.60 & 17.00 & 7.46 \\
\hline 100 & $50-50$ & 0.5 & 6.70 & - & - & 7.50 \\
\hline 100 & $20-80$ & 0.5 & 8.70 & - & - & 28.50 \\
\hline $70-30$ & 100 & 0.5 & 9.27 & 13.00 & 25.00 & 11.80 \\
\hline $70-30$ & $70-30$ & 0.5 & 8.45 & 11.13 & 18.35 & 18.00 \\
\hline 100 & 100 & 0.5 & 6.50 & 9.65 & 13.50 & 7.30 \\
\hline $20-80$ & 100 & 0.5 & 11.75 & 14.20 & 21.20 & 12.77 \\
\hline $50-50$ & 100 & 0.5 & 10.50 & 13.20 & 19.51 & 10.90 \\
\hline $80-20$ & 100 & 0.5 & 7.12 & 11.37 & 16.90 & 9.27 \\
\hline
\end{tabular}

Note: (a) $n=3, S D=7 \%$ except for 6 -nitrochysene: $9 \%$.

vi) Speed flow. A linear relationship exists between speed flow and pressure. Consequently correlated data were obtained here for different speed flows (Table 6 to 8 ). Therefore, it is not necessary to include this variable in the modeling of retention times when pressure is taken into account. On the other hand, although high heat friction is produced under high speed flows, especially for particles sizes under $5 \mu \mathrm{m}$; we use $5 \mu \mathrm{m}$ particles of size and a speed flow of ca. $0.5 \mathrm{ml} / \mathrm{min}$, in which case heat friction can be neglected [64].

vii) Temperature. The influence of this variable seems to be related to the physical properties of the mobile phase (especially the mobile phase viscosity). Since the effect of viscosity is included in the paper through the pressure inside the column for the different solvents studied, the effect of temperature is not considered.

In summary, we can state that the longest retention times were found using acetonitrile and methanol as mobile phases. If dipole moment of mobile phase is zero, high pressure entails shorter retention time (cyclohexane and hexane), whereas if the dipole moment of the mobile phase is higher, the retention times of PAHs and 6-nitrochrysene increase (Tables 6 and 7). When cyclohexane or hexane are used as mobile phase, the elution order of the target compounds seems to vary as a function of their respective molecular weight.
However, Table 6 shows shorter retention times for 6-nitrochryesene than for its parent PAH (chrysene) except when ethanol, cyclohexane and n-hexane are used as mobile phase. The shortest retention times for this nitro-PAH were obtained using dichloromethane, cyclohexane, and hexane, probably for the same reasons as for PAHs, namely, low dipole moment for these mobile phases. In the case of dichloromethane low dipole moment combined with low pressure gives rise to a very low interaction term between pressure and dipole moment, and consequently short retention time. Cyclohexane has no dipole moment, and consequently no interaction term between pressure and dipole moment; so high pressure means high work made by the system and short retention time. Nhexane provides retention time longer than cyclohexane, probably due to the lower pressure developed using n-hexane and consequently lesser work developed by the system. Otherwise, the longest retention times were obtained using acetonitrile and methanol whose dipole moments are high. It seems that combination of high pressure and high dipole moments implies long retention times as a consequence of a significant interaction between these variables. Although dipole moment of methanol is lower than that of acetonitrile, retention times of PAHs and 6-nitrochrysene are similar using either methanol or acetonitrile. This is probably due to higher viscosity of methanol and consequently higher 
pressure developed. However, unlike PAHs, retention time of 6- nitrochrysene varies only over a very short range (Tables 5 and next). The anomalous behaviour of 6-nitrochrysene compared to that of PAHs might be due to interactions among dipole 6-nitrochrysene molecules.

Table 6. Retention times of several PAHs and 6-nitrochrysene as a function of physical properties of the mobile phase (speed flow: $0.5 \mathrm{ml} / \mathrm{min}$ ).

\begin{tabular}{|c|c|c|c|c|c|c|c|}
\hline \multirow[t]{2}{*}{ Solvent } & \multirow{2}{*}{$\begin{array}{l}\text { Dipole } \\
\text { Moment }\end{array}$} & \multirow{2}{*}{$\begin{array}{l}\text { Viscosity } \times 10^{3} \\
\text { Pa.s } \\
\text { (Temperature) }\end{array}$} & \multirow{2}{*}{$\begin{array}{l}\text { Column } \\
\text { pressure } \\
\text { (bar) }\end{array}$} & \multicolumn{4}{|c|}{ Retention Time in min (a) } \\
\hline & & & & Naphthalene & Pyrene & Chrysene & $\begin{array}{l}\text { 6-nitro- } \\
\text { chrysene }\end{array}$ \\
\hline Acetonitrile & 3.44 & $0.369\left(25^{\circ} \mathrm{C}\right)$ & 18 & 6.50 & 7.50 & 9.65 & 7.30 \\
\hline Methanol & 2.87 & $0.584\left(20^{\circ} \mathrm{C}\right)$ & 23 & 6.40 & 7.89 & 10.00 & 7.83 \\
\hline Ethanol & 1.66 & $1.20\left(20^{\circ} \mathrm{C}\right)$ & 48 & 5.85 & 6.00 & 6.20 & 6.50 \\
\hline Dichloromethane & 1.14 & $0.423\left(25^{\circ} \mathrm{C}\right)$ & 22 & 6.05 & 6.15 & 6.20 & 6.10 \\
\hline Cyclohexane & 0 & $1.02\left(17^{\circ} \mathrm{C}\right)$ & 44 & 6.05 & 6.00 & 6.10 & 6.30 \\
\hline n-Hexane & 0 & $0.51\left(20^{\circ} \mathrm{C}\right)$ & 25 & 6.12 & 6.20 & 6.30 & 6.85 \\
\hline
\end{tabular}

Note: (a) $n=3, S D=7 \%$ except for 6 -nitrochysene which was $9 \%$, Dipole moments and viscosity data were obtained from Riddick and Bunger, 1970 [45].

Table 7. Retention times of several PAHs and 6-nitrochrysene as a function of physical properties of the mobile phase (speed flow: $0.25 \mathrm{ml} / \mathrm{min}$ ).

\begin{tabular}{|c|c|c|c|c|c|c|c|}
\hline \multirow[t]{2}{*}{ Solvent } & \multirow{2}{*}{$\begin{array}{l}\text { Dipole } \\
\text { Moment }\end{array}$} & \multirow{2}{*}{$\begin{array}{l}\text { Viscosity } \times 10^{3} \\
\text { Pa.s } \\
\text { (Temperature) }\end{array}$} & \multirow{2}{*}{$\begin{array}{l}\text { Column } \\
\text { pressure (bar) }\end{array}$} & \multicolumn{4}{|c|}{ Retention Time in min (a) } \\
\hline & & & & Naphtalene & Pyrene & Chrysene & $\begin{array}{l}\text { 6-nitro- } \\
\text { chrysene }\end{array}$ \\
\hline Acetonitrile & 3.44 & $0.369\left(25^{\circ} \mathrm{C}\right)$ & 10 & 12.80 & 13.50 & $\mathbf{1 7 . 5 3}$ & 14.30 \\
\hline Methanol & 2.87 & $0.584\left(20^{\circ} \mathrm{C}\right)$ & 14 & 12.20 & 13.72 & 17.88 & 14.76 \\
\hline Ethanol & 1.66 & $1.20\left(20^{\circ} \mathrm{C}\right)$ & 28 & 11.20 & 13.00 & 15.73 & 14.00 \\
\hline Dichloromethane & 1.14 & $0.423\left(25^{\circ} \mathrm{C}\right)$ & 11 & 12.80 & 12.90 & 14.64 & 13.50 \\
\hline Cyclohexane & 0 & $1.02\left(17^{\circ} \mathrm{C}\right)$ & 21 & 11.50 & 12.00 & 12.74 & 13.40 \\
\hline n-Hexane & 0 & $0.51\left(20^{\circ} \mathrm{C}\right)$ & 13 & 12.25 & 12.50 & 13.22 & 13.90 \\
\hline
\end{tabular}

Note: (a) $n=3, S D=5 \%$, except for 6-nitrochrysene: $7 \%$

As a conclusion, based on literature and on our own experience with RPLC, we thought that an interaction between dipole moment of the mobile phase and pressure inside the column could explain and predict the retention times of target analytes in $\mathrm{C}_{18}$-RPLC columns. On the other hand, possible repulsion among dipole molecules might be responsible for shortening the retention times of 6-nithrochrysene when low polarity solvents are used or when pressure lowers. This was our initial hypothesis.

3.1 Modeling of the retention dependence on the interaction mobile phase dipole moment-pressure

In order to evaluate the influence of the mobile phase dipole moment and the pressure on the retention times of the probe molecules (PAHs and 6-nitrochrysene), different solvents were used as mobile phase. As the solvents differ in viscosity, the pressure changes too, and consequently the simultaneous effect of both variables could be studied in an easy way by changing the mobile phase (Table 2). Dipole moments of the solvents ranged from 0 (hexane) to 3.44 (for acetonitrile) [65]. This range is wide since acetonitrile is the organic solvent with the highest dipole moment used in RPLC. Pressures ranged from 10 to 48 bar.

\subsubsection{Modeling of naphthalene Retention Times}

Retention times can be modeled using power functions as mentioned in paragraph 2.3. For Naphthalene, we obtained the following equation:

$$
R_{t}=\mu\left(P^{0.20}-1.60\right)-0.12 P+14
$$

where $R_{t}$ is the retention time (in $\min$ ), $P$ is the pressure (in bar), $\mu$ is the dipole moment (in Debye).

Equation 3 includes an interaction term (dipole moment interacts with pressure), one negative term of pressure and one constant. As we can see in Table 8 , 
the experimental data and the predicted values obtained by the model were in very good agreement for all the solvents. The shortest retention time was obtained by using cyclohexane or ethanol as mobile phase. These solvents have low dipole moment and high viscosity. Hence high pressures are developed inside the column, in that way decreasing retention times of naphthalene. Our study included also the researching of retention times of some PAHs on their solubilities in the mobile phase. Thus, the dipole moment of ethanol is 1.66 (Tables 6 and 7) and the molar solubility of naphthalene in this solvent is only 0.018 (Table 3), whereas molar solubility of naphthalene in dichloromethane is 0.33 (Table 3), i.e. 18 times higher. However, the retention time of naphthalene in dichloromethane is higher than in ethanol (Table 8). As for 6-nitrochrysene, solubility data do not account for the retention times. Naphthalene retention times are shorter than those of the other PAHs and 6-nitrochrysene. This is probably due to the lower molecular polarizability of naphthalene.

\subsubsection{Modeling of Pyrene Retention Times}

For pyrene, the following equation was obtained:

$$
R_{t}=\mu\left(P^{0.20}-1.35\right)-0.12 P+14
$$

where $R_{t}$ is the retention time (in $\min$ ), $P$ is the pressure (in bar), and $\mu$ is the dipole moment (in Debye). The retention time equation for pyrene is similar to that obtained for naphthalene, suggesting very similar retention mechanism for both of these PAHs. Again, good agreement between predicted and measured retention times was found (Table 8). The longest retention times were obtained by using acetonitrile and methanol as mobile phase, the shortest retention time was obtained using cyclohexane or ethanol, as in the case of naphthalene.

\subsubsection{Modeling of chrysene Retention Times}

For chrysene, we found the following power function:

$$
R_{t}=\mu\left(P^{0.35}-1.15\right)-0.06 P+14
$$

where $R_{t}$ is the retention time (in $\min$ ), $P$ is the pressure (in bar), and $\mu$ is the dipole moment (in Debye). Retention times for chrysene as a function of the mobile phase dipole moment and pressure inside the column are presented in Table 8. As we can be seen from this Table, interaction between pressure and dipole moment gains importance in the case of chrysene compared to lighter PAHs. The aforementioned coefficients of the variables vary from one PAH to another, depending on the molecular polarizability of each probe molecule. In general, the importance of interaction between pressure and dipole moment increases with increasing analyte's molecular polarizability in the case of polar solvents (see positive exponents in all the relationships). Otherwise, when non-polar solvents (cyclohexane and hexane) are used as mobile phase (cf. section 'results and discussion') high pressure entails high work developed by the system and consequently, shorter retention times.

\subsubsection{Modeling of 6-nitrochrysene Retention} time In the case of 6-nitrochrysene, we found the following relationship relating retention time, pressure, and mobile phase dipole moment:

$$
R_{t}=\mu^{2.5}\left(P^{0.10}-1.25\right)-0.01 P+14.00 \text { (6), }
$$

where $R_{t}$ is the retention time (in $\min$ ), $P$ is the pressure (in bar), and $\mu$ is the dipole moment (in Debye). As for the PAHs studied in previous paragraphs, the relationship obtained for 6nitrochrysene (6) includes an interaction term (dipole moment interacts with pressure), a negative term of pressure and a constant. In this case, the power of dipole moment is higher than for PAHs. Hence the dependence of 6-nitrochrysene retention time with interaction pressure-mobile phase dipole moment will gain importance at high pressure, but will lose importance at low pressures, as seen in previous sections. As we can see in Table 8, the experimental data and the predicted values obtained by the model were in very good agreement. As expected after examination of the data in Tables 6 and 7, the relationship between pressure and retention times is not trivial. However, the models constructed for PAHs or 6nitrochrysene revealed a strong interaction between the mobile phase dipole moment and pressure. Although only few solubility data for nitro-PAHs are available, it seems that they are more soluble in methanol and other polar solvents than PAHs [66]. However, as for PAHs, retention time for 6-nitrochrysene is higher in methanol than in low-dipole moment mobile phases. The constant term appearing in the equations might be indirectly related to total energy of the system. 
Table 8. Retention times of PAHs and 6-nitrochrysene as a function of dipole moment of the mobile phase and pressure inside the column (speed flow: $0.25 \mathrm{ml} / \mathrm{min}$ ).

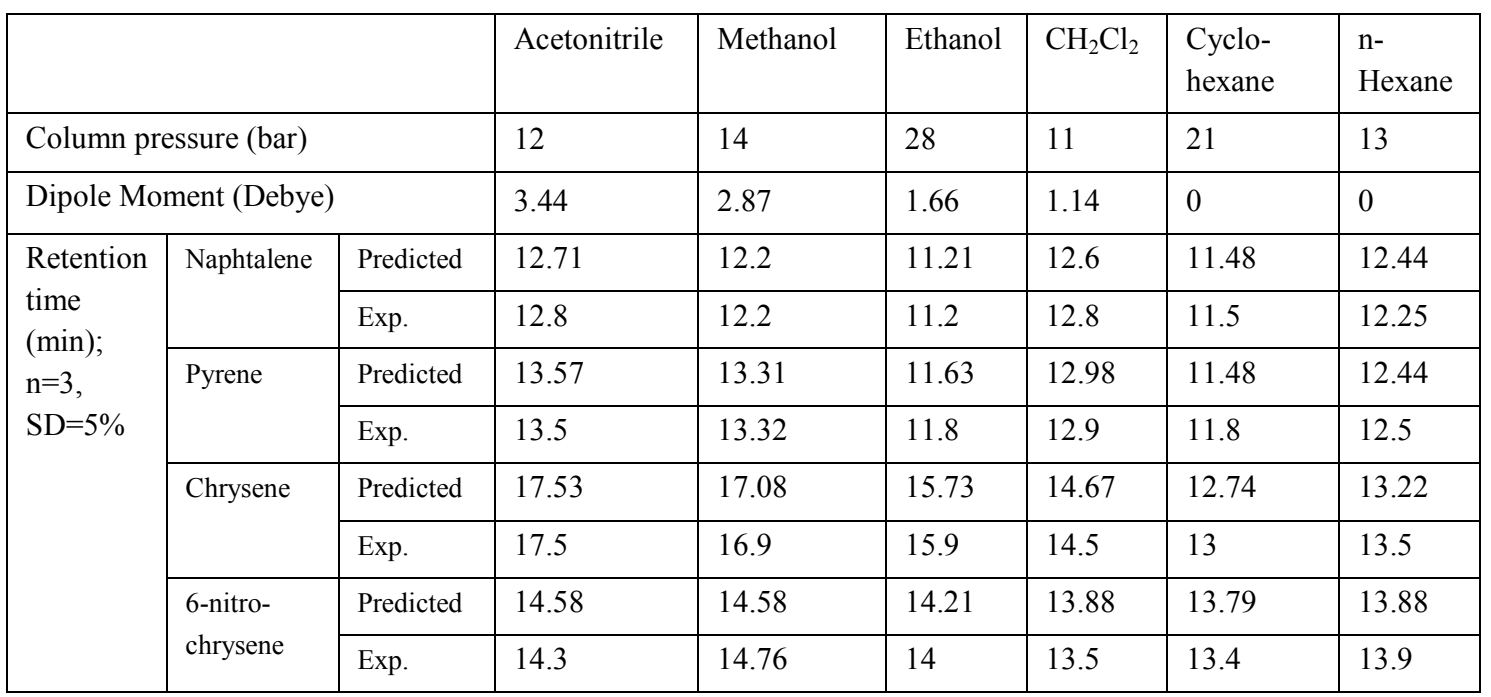

3.2. Revision of traditional approaches for RPLC mechanism So far, several thermodynamic approaches have been developed for explaining how the HPLC systems work. Most of the thermodynamics parameters have been obtained for gas-liquid interactions [14]. However, the magnitude of the parameters proposed cannot explain how the analytes (especially analytes with high medium polarizability) can remain inside RPLC column as long as 60 minutes (in the case of large PAHs under usual experimental conditions in RPLC) [1]. Some authors stated that RPLC is similar to classical liquid-solid chromatography for which interactions between solute and stationary phases are even weaker than in adsorption [2].

However,electromagnetophoretic measurements of adsorption forces of polystyrene micro particles (in water) on silica surfaces yielded values of adsorption forces of ca. $100 \mu \mathrm{N} / \mathrm{m}$ for $15 \mu \mathrm{m}$ diameter polystyrene particles and adsorption forces of ca. $80 \mu \mathrm{N} / \mathrm{m}$ for $20 \mu \mathrm{m}$ diameter polystyrene particles [67]. The authors concluded that the adsorption force was mainly governed by Van der Waals forces [68, 69]. These forces are not strong enough for explaining strong retentions in HPLC columns. We are going to prove that the retention in RPLC must be the consequence of forces much stronger than adsorptive forces.

i) The system work developed by RPLC devices at pressures of ca. 13-18 bar developed under usual experimental conditions (50\% ACN/Water, 0.25 $\mathrm{ml} / \mathrm{min}, \mathrm{T}=30^{\circ} \mathrm{C}$ ), can be calculated as follows:

SystemEnergy $=P \times V($ Pressure $\times$ Volume $)$, hence

SystemEnergy $=13 \mathrm{~atm} \times 1.5 \mathrm{ml} \times 0.001 \mathrm{l} / \mathrm{ml} \times 101 \mathrm{~J} / \mathrm{atml}=1.96 \mathrm{~J}$ where $1.5 \mathrm{ml}$ is the system volume, and 13 atm one of the lowest pressure values in RPLC. We should note that RPLC systems are not supposed to be used under 11 bar.

ii) On the other hand, the total energy of the system considered (mobile phase plus analyte) is given by expression (8):

$$
E=E_{c}+E_{g}+E_{p}+\Delta H_{s}+E_{v}
$$

That is, an energy balance including kinetic energy $\left(E_{c}\right)$, pressure energy $\left(E_{p}\right)$, gravitational energy $\left(E_{g}\right)$, solvation energy $\left(\Delta H_{s}\right)$, and energy of viscosity $\left(E_{v}\right)$.

$$
E=E_{p}+1 / 2 m v^{2}-m g-E_{v}+\Delta H_{s}
$$

where $m$ is mass, $g$ is gravity constant, and $v$ is speed flow.

As the energy developed by the system can be taken as the resultant of

$$
E_{p}+1 / 2 m v^{2}-m g-E_{v}
$$

only $\Delta H_{s}$ must be calculated. Taking into account that

$$
\log S=-\frac{\Delta H_{s}}{2.3 R T}+\text { cte } \quad[70,71,72]
$$

where $S$ is solubility, $\Delta H_{s}$ is the enthalpy of solvation, $R$ is the universal constant of gases, and $T$ is temperature in $K$. Cte can be calculated using solubility data at different temperatures (Table 9). For n-hexane, Cte $=2.52$. Thus, the energy of system-solvation is

$$
\Delta H_{s}=-2.3 R T \log S+2.52
$$

$$
\Delta H_{s}=20 \text {. }
$$


The quantity of naphthalene injected in the column is approximately: $2.6 \mathrm{ng}$ (130 ppb, $20 \mu \mathrm{L}$ injected). That is, $2.03 \times 10^{-11} \mathrm{~mol}$, and consequently $\Delta H_{s}$ would come $4.06 \times 10^{-10} \mathrm{~J}$. This is an energy certainly not significant compared to the total energy of the RPLC system and as a consequence, higher energies than simple solvation energies should be involved in the RPLC retention mechanism, as proved in previous paragraphs.

For checking out these conclusions, anthracene and phenanthrene retention times were studied. Anthracene and phenanthrene were chosen for this comparative study because of their very similar structure (i.e. the same number of aromatic rings) and their very different solubility in hexane. Cyclohexane solutions of anthracene and phenanthrene (in concentrations about $500 \mathrm{ppb}$ ) were individually prepared and injected in HPLC. Chromatographic runs were carried out using nhexane as mobile phase and a speed flow of 0.5 $\mathrm{ml} / \mathrm{min}$. No significant differences in retention times were found although the solubility of anthracene in n-hexane is 21 times lower than that of phenanthrene (Table 10).

Table 9. Solubility data for naphtalene in n-hexane as a function of temperature (NIST data:

http://srdata.nist.gov/solubility/sol detail.aspx?go Back $=$ Y\&sysID $=59$ 95) [108].

\begin{tabular}{|l|l|l|}
\hline T/K & Mole Fraction X1 & Mole Fraction X2 \\
\hline 282.05 & 0.063 & 0.937 \\
\hline 286.35 & 0.072 & 0.928 \\
\hline 289.55 & 0.084 & 0.916 \\
\hline 289.85 & 0.082 & 0.918 \\
\hline
\end{tabular}

Table 10. Comparison of retention times of anthracene and phenanthrene in RPC using n-hexane mobile phase (NIST data:

http://srdata.nist.gov/solubility/sol_detail.aspx?go Back $=$ Y\&sysID=59_95) [108].

\begin{tabular}{|l|l|l|}
\hline Compound & $\begin{array}{l}\text { Retention } \\
\text { Time in min } \\
\text { (a) }\end{array}$ & $\begin{array}{l}\text { Solubility } \\
\text { (molar fraction, } \\
\mathbf{2 9 8 K} \text { ) }\end{array}$ \\
\hline Anthracene & 12.70 & 0.0015 \\
\hline Phenanthrene & 12.80 & 0.0326 \\
\hline
\end{tabular}

Note: (a) $n=3$, Standard Deviation $=5 \%$

All these results confirm that solubility has no influence on retention times. As we have seen, solvation energy is a very small term compared to the system energy and retention times are not significantly different even if solubility differs greatly.

\subsection{The alternative hypothesis proposed}

To all the theories appearing in the literature, an alternative is proposed here. All the data from literature along with our conclusions seem to indicate that inside the column, an electric field is created, most likely by the vertical spontaneous alignment of dipoles (acetonitrile, water, methanol ...) on $\mathrm{C}_{18}$ chains surfaces which would behave as organic mono-layers [73, 74]. Only the alignment of dipoles in $\mathrm{C}_{18}$ chains and homogeneous polarization of silica particle could yield a global electric field [75] because even though dipoles can also be arranged or aligned on silica particles surface, this alignment would not be able to yield to electric field since the high symmetry of silica would generate a curl of polarization equal to zero. But is this alignment possible? Usually, only ferroelectric materials like barium titanate, give rise to spontaneous polari-zation due to the interaction among dipoles in the structure. But in the solid-liquid inter-phase, rupture of symmetry exists (like in $\mathrm{C}_{18}$-mobile phase inter-phase) and consequently spontaneous polarization of dipoles on the surface is possible [74]. As a matter of fact, recent studies in physics of dielectrics reported that not only interfacial polarization phenomena of organic molecular films on metallic surfaces are possible but also in inter-phase surfaces of very different dielectric constant (water-air) where breaking of symmetry occurs [74]. It mainly deals with dielectric polarization of rod-like molecules aligned to form mono-layers with symmetry $\mathrm{C}_{\infty}$ $[76,77,78,79,80,81,82]$. Each molecule is assumed to have a permanent dipole moment $\mu$ along its molecular long axis, and an anisotropic electronic polarizability, ie. the electronic polarizability $\alpha_{11}$ parallel to the molecular long axis, and $\alpha_{\alpha}$, perpendicular to the molecular long axis. Furthermore, the molecule has molecular secondorder susceptibility, $\alpha_{M}{ }^{(2)}$. Wagner et al. [83] consider the water surface and the monolayer film itself can be represented as an infinite plane. The dipole at the origin is facing on the water surface, and it tilts with an angle $\theta$. Molecular area decreases and increases as the monolayer films are compressed and expanded, respectively. These studies stated that high pressure avoids the tilt of polar molecules on the water surface, leading to increased polarization of the film [83]. Similar effects were shown by Manciu and Ruckenstein [51] who described the water dipoles alignment in vicinity of a surface as a phenomenon occurring at discrete positions. Stoylov [84] proposed a Maxwell-Wagner mechanism for explaining polarization of colloid particles with zero surface 
electric charge. In this case, the magnitude of polarization and its relaxation time depend exclusively on the difference in the dielectric properties of the particle and the medium (more precisely their complex permittivity, i.e. their dielectric constants and specific conductivities). It seems that this mechanism of polarization might prevail for slightly anisometric and/or heterogeneous micro-metric particles [84], like interphases $\mathrm{C}_{18}$-mobile phase in silica- $\mathrm{C}_{18}$ materials. On the other hand, if the electrical conductivity of organic films is sufficiently low (in fact, pure solvents such as water or ethanol are dielectrics, that is insulating materials [85]), the establishment of thermodynamic equilibrium over the whole bulk region of the prepared organic materials is very difficult due to long-lasting dielectric relaxation phenomena [86]. As a result, energy level alignment is restricted at inter-phase and the concept of surface Fermi-level becomes more helpful. This means that theoretical and experimental approaches based on dielectric physics are also very important to study nano-interfacial phenomena of organic materials [74].

Regarding dipole mobile phases included in this paper, acetonitrile (as well as methanol or ethanol) can be considered a rod-like polar molecule. Anisotropic response was analyzed using a separation scheme that allows a decomposition of the total response in terms of orientation and collision-induced effects as introduced by Elola and Ladanyi [87]. This solvent presents a collective polarizability induced by intermolecular interactions where orientational dynamics is the main contribution to the anisotropic response, although intermolecular collision-induced (or translational) effects are important [87].

In addition to the spontaneous polarization at the interface solid/liquid, silica spherical particle can also undergo polarization through the external electric field created by the spontaneous alignment of dipoles in the $\mathrm{C}_{18}$ chains. Although commercial amorphous silica used in HPLC [88] (isotropic and lineal material) is more polarisable and covalent than similar ionic oxides [88] when it is derivatized with $\mathrm{C}_{18}$ chains, its dipole moment is expected to fall because of dipole moment silica- $\mathrm{C}_{18}$ bound become zero [90]. As a consequence, the polarizability of the material will be low and the created electric field too [90] an electric field might be created anyway because a sphere uniformly polarized can create an electric field equal to one dipole situated in the centre of the particle whose electric moment was $\zeta P$, where $\zeta$ is the volume of silica particle and $P$ is the polarization [91]. On the other hand, due to the alignment of dipoles on the $\mathrm{C} 18$ chains, this electric field can be reinforced.

\subsection{Retention mechanism in RPC through spontaneous surface polarization}

Although this is the first time a resultant electric field created by spontaneous polarization in surfaces is proposed to be responsible for retention in RPLC, papers about interactions between molecules, such as endo-fullerenes, and electric fields have been already published [92]. In addition to this, adsorption of neutral molecules on either positively or negatively electrochemically polarized electrode surface was recently carried out by Conway and Niu [93]. The authors emphasized that $\mathrm{C}$ materials (except diamond) can be studied as metals for which the electrochemical thermodynamics of substitution-adsorption is well understood. The potential of adsorption will arise from the field-effects in the electrode/solution interphase and it depends on the difference of dipole moments and polarizabilities of the analyte (or adsorbate) and co-adsorbed solvent molecules, as established in studies of adsorption on $\mathrm{Hg}$-drop electrode [94, 95]. Adsorption and electrosorption of heterocyclic compounds seem to be related to the replacement of pre-adsorbed and field-oriented solvent mole-cules by oriented adsorbates [93, 96, 97]. Accord-ing to these studies, high difference of dipole moments and polarizabilities between adsorbate and co-adsorbed solvent molecules are desirable. Adsorption of molecules on the RPLC surface is highly dependent on quadratic hyperpolarizeability of these molecules [98], defined as:

$$
\beta=(3 \gamma \mu / \alpha)
$$

Where $\gamma$ and $\alpha$ are two constants depending on the geometry of the compound, and $\mu$ is the dipole moment in absence of external interactions [99]. $\beta$ increases when the molecule presents a strong non linear behavior. As asymmetry is one of conditions that must be fulfilled by the molecule for non linear behavior, organic compounds with $\pi$ electrons (easily polarizable) can show nonlinear behavior if asymmetry occurs [100, 101]. This could explain why the asymmetric isomers of pyrene such as chrysene or benzo(a)anthracene are retained stronger on $\mathrm{C}_{18}$ compared to pyrene (symmetric). Also Jinno and Kawasaki [61] stated that there is a high correlation between retention times for PAHs in RPLC and their molecular polarizability.

Regarding elution solvents, recent conclusions on superheated water as mobile phase, agree with our results. Superheated water was found to elute solutes from glass beads, Florisil, and alumina, whereas higher temperatures were required for $\mathrm{C}_{18^{-}}$ 
silica [102]. Since dielectric constant of water diminishes with increasing temperature [103] and retention strengths on glass, florisil, and alumina are smaller than on $\mathrm{C}_{18}$-silica (because of shorter differences of dielectric constant between particles and mobile phase in glass, florisil and alumina cases than those between $\mathrm{C}_{18}$-silica and mobile phases as acetonitrile), higher temperatures than those required for eluting analytes from glass, florisil, and alumina particles should be required for eluting analytes from $\mathrm{C}_{18}$-silica particles. This result is totally consistent with our hypothesis based on electromagnetic forces created by spontaneous alignment of mobile phase dipoles on the stationary surface.

By application of polarization surface theory, the reason why the adsorption equilibrium constant (K) does not increase for carbon contents above around $10 \%$ could be also easily understood. As a matter of fact, as early as 1934, Arkel and Snoek [104] reported on the dielectric behavior of concentrated solutions of dipole substances. They found that the polarization shows a marked decrease with increasing dipole concentration. Several decades later, Kliem and Fuhrmann [105] deduced that such behavior can be explained by nonlinear electrostatic dipole interaction. Computer simulation shows that the electrostatic dipole-dipole interaction in disordered systems of bidirectional dipoles leads to a decrease of the static polarization of the dielectric [105]. Similar conclusions were drawn by $\mathrm{Hu}$ et al. [106] for surface ions on a spherical colloidal particle.

A similar phenomenon might occur on $\mathrm{C}_{18}$-silica surfaces. In fact, simple physical calculations drive to the same conclusions: resultant electric field in the line bonding two dipoles diminishes when the distance between dipoles diminishes [107]. This could be the reason why too high density of $\mathrm{C}_{18}$ chains coverage is not recommended. An equilibrium between polarization surface (and consequently electric field) and electrostatic repulsion forces must be considered.

Our hypothesis would explain a wide range of phenomena in RPLC, i.e.:

1. Why high pressures are fundamental in RPLC. As we saw along the text, high pressure could avoid the tilt of rod like dipoles (molecules of mobile phase) and consequently it would favor the retention in this kind of columns.

2. Why it is not possible to separate of PAHs by using non-polar solvents in RPLC. In fact, nonpolar solvents like $n$-hexane have very low dipole moment and they cannot create electric fields.

3. Why compounds with large number of aromatic rings are highly retained in RPLC in comparison with the lightest ones. This could be because the higher PAHs exhibit higher molecular polarizability. On the other hand, our hypothesis could explain why the asymmetric isomers of pyrene such as chrysene or benzo(a) anthracene are stronger retained in $\mathrm{C}_{18}$ compared to pyrene (symmetric).

4. Why a RPC column can remain polarized during a very long time after switching off the system.

5. Why dipole molecules (nitroPAHs) show a behavior so different from that of neutral analytes (PAHs) into a RPLC column. The same conclusions could be drawn for other dipole molecules according to literature. Loeser [60] found also shorter retentions times for dipole molecules, whereas the retention times of anions (i.e. $\mathrm{Cl}^{-}$) was comparatively higher. Finally, Gotkiewicz [108] had also reported shorter retention times for 1,4dihydroxybenzene, 1,3-dihydroxybenzene and 1,2dihydroxybenzene than for hydroxybenzene in a RPLC system using different gradients of methanol-water and a $\mathrm{C}_{18}$ reversed-phase. As we can see, under usual RPLC experimental conditions retention times are shorter for dipole than for monopoles of similar structure and molecular weight.

6. Why derivatized silica surfaces are highly recommended for analytical separation of neutral compounds (in this case, PAHs) by RPLC. In fact, studies on dielectric physics suggest that the solid phase polarization increases with increasing dielectric constants differences between the solid phase and the liquid bulk [84].

7. Why above a certain percentage of $\mathrm{C}$ in silica particles, retention times do not improve. In fact, $\mathrm{C}_{30}$ chains do not improve the retention in RPLC columns in comparison to $\mathrm{C}_{18}$ columns. As we saw in previous paragraphs, papers on the dielectric behavior of concentrated solutions of dipole substances demonstrated that the polarization shows a marked decrease with increasing dipole concentration most likely due to nonlinear electrostatic dipole interaction.

7. Why retention times are a function of temperature. Temperature affects the dielectric properties of surfaces and the viscosity of the mobile phase.

8. Why elution order in RPLC does not depend on the solubility of the target analyte.

9. Why an aromatic compound (or in general, compounds with conjugated double bonds) can be retained inside an analytical column during a time as long as 1 hour even working at very high pressures. Dipole-dipole interactions, dipoleinduced dipole interactions, instantaneous dipole- 
induced dipole interactions, hydrogen bonding interactions, electron pair donor-electron acceptor interactions cannot explain the strong retentions observed in RPLC.

10. Why the conditioning steps are so important in a RPLC determination.

11. This work considers neutral compounds and only one dipole molecule (6-nitrochrysene) although the theory could be extended to ions and acid/basic compounds if electrostatic repulsions among target analytes are taken account.

\section{Conclusions}

This paper reports original conclusions about RPLC retention of non-polar and dipolar organic compounds. Although not significant and/or evident influence of the pressure on the PAHs retention times was observed for any of the mobiles phases considered in this paper, we found that pressure influences the retention time of 6nitrochrysene using very polar mobile phases. On the other hand, according to the mathematical equations constructed for modeling the retention times of probe molecules in a RPLC column, interaction between dipole moment of the mobile phase and pressure inside the column exists. This interaction increases upon increase of the probe molecule polarizability, and accounts on most of the phenomena observed in RPLC.

Although future work would be need for drawing definitive conclusions, evidence of an electric field created by spontaneous vertical alignment of mobile phase dipoles on $\mathrm{C}_{18}$-silica stationary phase particles exists.

\section{Acknowledgements}

Partial financial support from European Space Agency (ESA) through the contract 15091/01/NL/SH-CCN No. 002, MAP Project numbers AO-99-001 AO-99-085, is gratefully acknowledged. We also thank Professor Elisabeth Bosh from Barcelona University (Spain), Dr. Graciela de Armas from Balearic Islands University (Spain) and Santiago Pais for bibliographic assistance and Dr. Pascale Gillon, and Claude Fougère from CNRS-ICARE, Orléans (France) for technical support. Finally, we are grateful to two anonymous referees for having helped us to improve the paper.

\section{References}

[1] A. Andrade-Eiroa, V. Leroy, P. Dagaut, Y. Bedjanian, Chemosphere 78 (2010) 1342. http://dx.doi.org/10.1016/j.chemosphere.2010. $\underline{01.005}$
[2] J.H. Knox, A. Pryde, J. Chromatogr. 112 (1975), 289.

[3] Y.D. Men, D.B. Marshall, Anal. Chem. 62 (1990) 2606.

http://dx.doi.org/10.1021/ac00222a016

[4] T.L. Chester and J.W. Coym, J. Chromatogr. A 1003 (2003) $101 . \quad$ http://dx.doi.org/ 10.1016/S0021-9673(03)00846-X

[5] P. T. Ying, J.G. Domy, K.A. Dill, Anal. Chem. 612 (1989) 540.

[6] C.A. Doyle T.J. Vickers, C.K. Mann, J.G. Dorsey, J. Chromatogr. A 877 (2000) 25.

[7] P.W. Carr, J. Li., A. Dallas, D.I. Eikens and L.C. Tan, J. Chromatogr. A 656 (1993) 113. http://dx.doi.org/10.1016/00219673(93)80800-N

[8] L. Zhang, L. Sun, J.I. Siepmann, M.R. Schure, J. Chromatogr. A, 1079 (2005) 127.

http://dx.doi.org/10.1016/j.chroma.2005.03.124

[9] Cs. Horváth, W.R. Melander and I. Mólnar, J. Chromatogr. A 125 (1976) 129.

[10] K.A. Dill, J. Phys. Chem. 91 (1987) 1980. http://dx.doi.org/10.1021/j100291a060

[11] J.G. Dorsey and K.A. Dill, Chem. Rev. 89 (1989) 331.

http://dx.doi.org/10.1021/cr00092a005

[12] R. Tijssen, P.J. Shoenmakers, M.R. Böhmer, L.K. Koopal and H.A.H. Billiet, J. Chromatogr. A 656 (1993) 135.

http://dx.doi.org/10.1016/0021-9673(93)80801-E

[13] C.D. Wick, J.I. Siepmann and M.R. Schure, Anal. Chem. 76 (2004) 2886. http://dx.doi.org/10.1021/ac0352225

[14] J.L. Rafferty, J.I. Siepmann and M.R. Schure, Fluid Phase Equilibria, 290 (2010) 25. http://dx.doi.org/10.1016/j.fluid.2009.10.010

[15] C.F. Poole, The Essence of Chromotography, Elsevier, Amsterdam, The Netherlands, 2003, chap. 4, pp. 267-413.

[16] D.E. Martire, R.E. Boehm, J. Phys. Chem. 87 (1983) 1045. http://dx.doi.org/10.1021/j100229a025

[17] R.L. Hansen, M. Harris, Anal. Chem. 67 (1995) 492. http://dx.doi.org/10.1021/ac00099a003

[18] K.B. Sentell, D. M. Bliesner, S.T. Shearer, in Chemically Modified Surfaces, ed. J. J. Pesek and IE Leigh, Royal Society of Chemistry, Cambridge, UK, 1994, p. 190.

[19] N.S. Bahadur, W.Y. Shiu, D.G.B. Boocock, D. Mackay, J.Chem.Eng.Data 42 (1997) 685. http://dx.doi.org/10.1021/je970020p 
[20] P.L. Zhu Chromatographia 21 (1986) 229.

[21] R. B. Marcel, L.K. Koopal, Robert Tijssent, J. Phys. Chem. 95 (1991) 6285.

[22] C.H. Lochmüller, M.L. Hunnicutt, J.F. Mullaney, J. Phys. Chem. 89 (1985) 5770. http://dx.doi.org/10.1021/j100272a039

[23] C. Yan, D.E. Martire, J. Phys. Chem. 96 (1992) 3505.

http://dx.doi.org/10.1021/j100187a060

[24] F. Gritti, G. Guiochon, J. Chromatogr. A 1115 (2007) 85.

http://dx.doi.org/10.1016/j.chroma.2007.04.024

[25] M. R. Bohmer, R Tijssen, L.K Koopal, J. Phys. Chem. 95 (1991) 6285. http://dx.doi.org/10.1021/j100169a041

[26] K. Miyabe, G. Guiochon, Anal. Sci. 17 (2001) 209. http://dx.doi.org/10.2116/analsci.17.209

[27] F. Griti, G. Guiochon, Chem. Engineer. Sci. 61 (2006) 7636.

http://dx.doi.org/10.1016/j.ces.2006.08.070

[28] F. Gritti and G. Guiochon, J. Chromatogr. A 1115 (2006) 142.

http://dx.doi.org/10.1016/j.chroma.2006.02.095

[29] F. Gritti and G. Guiochon, J. Chromatogr. A, 995 (2003) 37.

http://dx.doi.org/10.1016/S00219673(03)00489-8

[30] F. Gritti, A. Felinger, G. Guiochon, J. Chromatogr. A 1017 (2003) 45.

http://dx.doi.org/10.1016/S00219673(03)01285-8

[31] T.S. Reid, R.A. Henry, Am. Lab. 31 (1999) 24.

[32] T.H. Walter, P. Iraneta, M. Capparella, J. $\begin{array}{lllll}\text { Chromatogr. A } & 1055 \quad \text { (2005) } & 177 .\end{array}$ http://dx.doi.org/10.1016/j.chroma.2005.04.03 9

[33] Y. Kazakevich and Y. El'tekov, Russ. J. Phys. Chem. 54 (1980) 83.

[34] J.E. Pemberton, M. Ho, C.J. Orendorff, M.W. Ducey, J. Chromatogr. A 913 (2001) 243. http://dx.doi.org/10.1016/S00219673(01)00569-6

[35] K.G. Miller, C.F. Poole, J. High Resolut. Chromatogr. 17 (1994) 125.

http://dx.doi.org/10.1002/jhrc.1240170302 http://dx.doi.org/10.1002/poc.610071205

[36] C.F. Poole, S.K. Poole, D.S. Seibert, C.M. Chapman, J. Chromatogr. B 689 (1997) 245. http://dx.doi.org/10.1016/S03784347(96)00282-4
[37] M.H. Abraham, H.S. Chadha and A.J. Leo, J. Chromatogr. A 685 (1994) 203.

http://dx.doi.org/10.1016/00219673(94)00686-5

[38] A.Wang, L.C. Tan, P.W. Carr, J. Chromatogr. A 848 (1999) 21.

http://dx.doi.org/10.1016/S0021-9673(99)00464-1

[39] D. Bolliet, C. F. Poole, Analyst 123 (1998) 295.http://dx.doi.org/10.1039/a705617f

[40] R. Kaliszan, Structure and Retention in Chromatograpy: A chemometric approach, Harwood Academic Publisher, Amsterdam, 1997, chap. 4, pp. 19-22.

[41] J.R. Torres-Lapasió, M.J. Ruiz-Ángel, M.C. García-Alvárez-Coque, J. Chromatogr. A 1166 (2007) 85 .

http://dx.doi.org/10.1016/j.chroma.2007.07.081

[42] C.F. Poole, W.Kiridena, C.de Kay, W.W. Koziol and R.D. Rosencrans, J. Chromatogr. A 115 (2006) 133.

[43] S. K. Kulkarin Jatkar, Nature 153 (1944) 222. http://dx.doi.org/10.1016/j.chroma.2006.02.089

[44] G. Srinivasan, L. Sander, K. Muller, Anal. Bioanal. Chem. 384 (2006) 514.

http://dx.doi.org/10.1007/s00216-005-0161-z

[45] G. Srinivasan, C. Meyer, N. Welsch, K. Albert, K. Müller, J. Chromatogr. A 1113 (2006) 45.

http://dx.doi.org/10.1016/j.chroma.2006.01.113

[46] G. Srinivasan, K. Müller, J. Chromatogr. A 1110 (2006) 102.

http://dx.doi.org/10.1016/j.chroma.2006.01.095

[47] L. Hansen, J.M. Harris, Anal. Chem. 68 (1996) 2879.

http://dx.doi.org/10.1021/ac960119j

[48] L.C. Sander, M. Pursch, S. A. Wise, Anal. Chem. 71 (1999) 4821. http://dx.doi.org/10.1021/ac9908187

[49] H.J. Egelhaaf, D. Oelkrug, M. Pursch, K. Albert, J. Fluoresc. 5 (1997) 311. http://dx.doi.org/10.1023/A:1022534110945

[50] L.C. Sander, K.A. Lippa, S.A. Wise, Anal. Bioanal. Chem. 382 (2005) 646. http://dx.doi.org/10.1007/s00216-005-3127-2

[51] M. Manciu, E. Ruckenstein, Langmuir 21 (2005) 11749. http://dx.doi.org/10.1021/la051802g

[52] S.Kayillo, G.R. Dennis, R.A Shalliker, J. Chromatogr. A 1126 (2006) 283. http://dx.doi.org/10.1016/j.chroma.2006.04.054

[53] Polarizabilities and dipole moments of 
benzaldehyde, benzoic acid and oxalic acid in polar and nonpolar solvents, Nalan Tekin, Mustafa Cebe, Celik Tarımcı, Chemical Physics 300 (2004) 239.

[54] P. Szabelski, A. Cavazzini, K. Kackmarski, X. Liu, J. Van Horn and G. Guiochon, J. Chromatogr. A 950 (2002) 41.

http://dx.doi.org/10.1016/S0021-9673(01)01614-4

[55] S.H. Chen and C.W. Li, J. Chromatogr. A 1023 (2004) 41.

http://dx.doi.org/10.1016/i.chroma.2003.09.057

[56] N. Tanaka, T. Yoshimura, N. Araki, J. Chromatogr. A 406 (1987) 247. http://dx.doi.org/10.1016/S00219673(00)94033-0

[57] M.M. Fallas, U.D. Neue, M.R. Hadley, D.V. McCalley, J. Chromatogr. A 1217 (2010) 276. http://dx.doi.org/10.1016/j.chroma.2009.11.041

[58] A.V. Avdeenkov, J. L. Bohn, Phys. Rev. Lett. 90 (2003) 043006/1-4.

[59] L. Vaidman, American J. Phys. 58 (1990) 978. http://dx.doi.org/10.1119/1.16260

[60] E. Loeser, J. Chromatogr. Sci. 46 (2008) 45.

[61] Y. Miura, S. Kimura, S. Kobayashi, M. Iwamoto, Y. Imanishi, J. Umemura, Chem. Phys. Lett. 315 (1999).

[62] K. Jinno, K. Kawasaki, Chromatographia 18 (1984) 103.

http://dx.doi.org/10.1007/BF02268467

[63] A. Andrade-Eiroa, P. Diévart, P. Dagaut, Talanta 81 (2010) 265.

http://dx.doi.org/10.1016/j.talanta.2009.11.068

[64] K. Kaczmarsky, F. Gritti, G. Guiochon, J. Chromatogr. A 1177 (2008) 92.

http://dx.doi.org/10.1016/j.chroma.2007.11.009

[65] J.A. Riddick and W.B. Bunger, in Organic Solvents Physical. Properties and Methods of Purification, Wiley-Interscience, New York, 3rd edition, 1970, vol. 2, pp. 1-200.

[66] IARC. 1989. Diesel and Gasoline Engine Exhausts and Some Nitroarenes. IARC Monographs on the Evaluation of Carcinogenic Risk of Chemicals to Humans, vol. 46. Lyon, France: International Agency for Research on Cancer, p. 458.

[67] Y. Iiguni, H. Watarai, Bull. Chem. Soc. Japan 79 (2006) 47. http://dx.doi.org/10.1246/bcsj.79.47

[68] M.I. Saleh, F. Adam, J. Am. Oil Chemists' Soc. 71 (1994) 1363. http://dx.doi.org/10.1007/BF02541356

[69] Y. Byrak, Microporous and Mesoporous Materials 87 (2006) 203. http://dx.doi.org/10.1016/j.micromeso.2005.08.009

[70] D. Patterson and Sheldon, Trans Faraday Soc. 55 (1959) 1254.

http://dx.doi.org/10.1039/tf9595501254

[71] W. Jost, in: Diffusion in solids, liquids, gases, Academic Process Inc. Publishers, 1960, p. 174.

[72] J. Cegarra, P. Puente, Textile Res. J. 37 (1967) 343.

http://dx.doi.org/10.1177/004051756703700502

[73] M. Iwamoto, T. Manaka, Anal. Chim. Acta 568 (2006) 65.

http://dx.doi.org/10.1016/j.aca.2005.12.018

[74] M. Iwamoto, T. Manaka, Colloids and Surfaces A: Physicochem. Eng. Aspects, 284 (2006) 29.

http://dx.doi.org/10.1016/j.colsurfa.2005.10.075

[75] A. Molitón, Basic Electromagnetism and materials, Springer, First Ed., New York, 2006, chap. 2, pp. 39-82.

[76] A. Tojima, T. Manaka, M. Iwamoto, J. Chem. Phys. 115, 19 (2001) 9010.

[77] A. Saupe, Z. Naturforsch A, 19A (1964) 161.

[78] V. Tsvetkov, Acta Physicochim. URSS 16 (1942) 132.

[79] A.V. Zakharov, M. Iwamoto, Phys. Review E 66 (2002) 061605, 1-7.

[80] S. Chandrasekhar, Liquid Crystals, Cambridge University Press, London, 1977.

[81] P.G. de Gennes in The physics of Liquid Crystals, Clarendon, Oxford, 1974, pp. 289292.

[82] M. Iwamoto, T. Manaka, Proc. Int. Symp. Super-Functionality Organic Devices IPAP Conferences 92 (2002) 7306.

[83] R. Wagner T. Yamamoto, T. Manaka, and M. Iwamoto, Review of Scientific Instruments, 76 (2005) 083902.

[84] S.P. Stoylov, Colloids and SurfacesB: Biointerfaces, 56 (2007) 50.

http://dx.doi.org/10.1016/j.colsurfb.2006.11.016

[85] A. Stengele S. Rey, H. Leuenberger, Int. J. Pharm., 225 (2001) 123.

[86] V. Kestelman, L. Pinchuk and V. Goldade, Electrets in engineering, 2nd Ed., Kluwer Academic Publishers, Boston London, 2000, chap. 1, 2-7.http://dx.doi.org/10.1007/978-1$\underline{4615-4455-5}$

[87] M.D. Elola, B.M. Ladanyi, J. Chem. Phys. 122 (2005) 22.

[88] J.S. Fritz, Analytical Solid Phase Extraction, Ed. John Wiley, New York, 1999, pp. 1-28. 
[89] N. Sahai, Environ. Sci. Technol. 36 (2002) 445. http://dx.doi.org/10.1021/es010850u

[90] G.N. Kartsev, I.Yu.Kokoreva, Ya.K. Syrkin, V.F. Mironov, E.A. Chernyshev, J. Struct. Chem. 6 (1965) 287. http://dx.doi.org/10.1007/BF00745959

[91] H. Gié, J.P. Sarmant, Electromagnétisme, Technique et documentation (Lavoisier), Paris, 1985, vol. 2, chap. 21-22, pp. 141-163.

[92] Y.K. Golikov, L.N. Gall, N.R. Gall, Bull. Russ. Academ. Sci. Phys. 62 (1998) 1602.

[93] B. E. Conway, J. Niu, Z. Phys. Chem. 218 (2004) 709.

http://dx.doi.org/10.1524/zpch.218.6.709.33451

[94] R. Parsons, Trans. Faraday Soc. 55 (1959) 999. http://dx.doi.org/10.1039/tf9595500999

[95] B.E. Conway, R.G. Barradas, Electrochim. Acta 5 (1961) 319.

http://dx.doi.org/10.1016/0013-4686(61)90005-6

[96] R.J. Watts-Tobin, N.F. Mott, Electrochim. Acta 4 (1961) 79.

http://dx.doi.org/10.1016/0013-4686(61)80009-1

[97] J. O’M. Bockris, M.A.V. Devanathan, K. Müller, Proc. Roy. Soc.London, Ser. A 274 (1963) 55.

[98] D.R. Kanis, M.A. Rather, T.J. Marks, Chem. Rev. 94 (1994) 195.

http://dx.doi.org/10.1021/cr00025a007

[99] J.R. Herance-Camacho, Ph D Dissertation, Reactivos ocluidos en aluminosilicatos. Reactividad y comportamiento en óptica no lineal, University Autonoma of Barcelona
(Spain), 2005.

[100] K.B. Everard, L.E. Sutton, J.Chem.Soc, (1951) 2818. http://dx.doi.org/10.1039/jr9510002818

[101] B.F. Levine, C.G.Betea, J.Chem.Phys. 63 (1975) 2666. http://dx.doi.org/10.1063/1.431660

[102] Y. Yang, M. Belghazi, A. Lagadec, D.J. Miller, S.B. Hawthorne, J. Chromatogr. A 810 (1998) 149. http://dx.doi.org/10.1016/S00219673(98)00222-2

[103] J.B. Hamelin, Mehl, M.R. Moldover, Int. J. Thermoph. $\quad 19 \quad$ (1998) 1359. http://dx.doi.org/10.1023/A:1021979401680

[104] A.E Van Arkel, Snoek J.L., Trans. Faraday $\begin{array}{llll}\text { Soc. } & 160 & \mathbf{( 1 9 3 4 )} & 707 .\end{array}$ http://dx.doi.org/10.1039/tf9343000707

[105] H. Kliem, P. Fuhrmann, Electrical Insulation and Dielectric Phenomena, 1989. Annual Report, Conference on 29 (1989) 321. [106] V. K. Hu, K. E. Blackmon, Int. J. Modern Phys. B 10 (1996) 3219.

http://dx.doi.org/10.1142/S0217979296001665

[107] C. Paul, S. Nasar Introduction to electromagnetic fields McGraw-Hill, 2nd. Ed., 1987, 34-45.

[108] W. Gotkiewicz, Chromatographia 14 (1989) 11.

[109] NIST data:

http://srdata.nist.gov/solubility/sol_detail.aspx ?goBack $=$ Y\&sysID $=5995$ 